\title{
Interaction effects of aging, word frequency, and predictability on saccade length in Chinese reading
}

\author{
Zhi Fang Liu ${ }^{\text {Corresp., Equal first author, } 1 \text {, Wen Tong }}{ }^{\text {Equal first author, } 2}$, Yong Qiang Su ${ }^{3}$ \\ 1 Department of Psychology, Hangzhou Normal University, Hang Zhou, China \\ 2 Department of Psychology, Shanxi Normal University, Lin Fen, China \\ 3 Department of Psychology, Beijing Normal University, Bei Jing, China \\ Corresponding Author: Zhi Fang Liu \\ Email address: 20171046@hznu.edu.cn
}

Background: It was well known that age has an impact on word processing (word frequency or predictability) in terms of fixating time during reading. However, little is known about whether or not age modulates these impacts on saccade behaviors in Chinese reading (i.e. length of incoming/outgoing saccades for a target word). Methods: Age groups, predictability, and frequency of target words were manipulated in the present study. A larger frequency effect on lexical accessing (i.e., gaze duration) and on context integration (i.e., go-past time, total reading time), as well as larger predictability effects on data of raw total reading time, were observed in older readers when compared with their young counterparts. Results: Effect of predictability and frequency on word skipping and re-fixating rate did not differ across the two age groups. Notably, reliable interaction effects of age, along with word predictability and/or frequency, on the length of the first incoming/outgoing saccade for a target word were also observed. Discussion: Our findings suggest that the word processing function of older Chinese readers in terms of saccade targeting declines with age. 


\section{Interaction effects of aging, word frequency, and} 2 predictability on saccade length in Chinese reading

3

\section{Zhi Fang Liu ${ }^{1}$, Wen Tong ${ }^{2}$, Yong Qiang $\mathrm{Su}^{3}$}

${ }^{1}$ Department of Psychology, Hangzhou Normal University, Hangzhou, Zhejiang Province, China

${ }^{2}$ Department of Psychology, Shanxi Normal University, Linfen, Shanxi Province, China

${ }^{3}$ Department of Psychology, Beijing Normal University, Beijing, China

Corresponding Author:

Zhi Fang Liu $^{1}$

No.2318, Yuhangtang Rd, Hangzhou, Zhejiang, Zhejiang Province, 311121, P. R. China Email address: lzhf2008@163.com

\section{Abstract}

Background: It was well known that age has an impact on word processing (word frequency or predictability) in terms of fixating time during reading. However, little is known about whether or not age modulates these impacts on saccade behaviors in Chinese reading (i.e. length of incoming/outgoing saccades for a target word).

Methods: Age groups, predictability, and frequency of target words were manipulated in the present study. A larger frequency effect on lexical accessing (i.e., gaze duration) and on context integration (i.e., go-past time, total reading time), as well as larger predictability effects on data of raw total reading time, were observed in older readers when compared with their young counterparts.

Results: Effect of predictability and frequency on word skipping and re-fixating rate did not differ across the two age groups. Notably, reliable interaction effects of age, along with word predictability and/or frequency, on the length of the first incoming/outgoing saccade for a target word were also observed.

Discussion: Our findings suggest that the word processing function of older Chinese readers in terms of saccade targeting declines with age.

\section{Introduction}

It has been well documented that word processing may vary among young and older adult readers of western languages, such as English and German. Specifically, recent evidence has revealed a subtle decline in word identification among older adults when they read; that is, older adults spend more time fixating on target words, and regress to them more often than younger readers (Kemper, Crow \& Kemtes, 2004; Laubrock, Kliegl, \& Engbert, 2006; Rayner, Castelhano, \& Yang, 2009, 2010; McGowan, White, Jordan, \& Paterson, 2014; Whitford \& Titone, 2017; Paterson, McGowan, \& Jordan, 2013a, 2013b, 2013c). Studies have also revealed 
40 that frequency and predictability effects, which are closely related to how easily any word can be processed, are also impacted by aging. For example, Kliegl et al. (2004) revealed that older readers yielded larger frequency effects, younger readers tended to skip more predictable words, and older adults re-fixated less on such words while reading in German. Rayner et al. (2006) also reported larger frequency effects for native older adult English readers than young adult readers; however, they failed to observe differences in predictability effects between the two age groups. Recently, Steen-Baker et al. (2017) reported a different contextual sensitivity in the regression patterns of older readers. A study conducted by Choi et al (2017) revealed larger predictability effects on fixation time measures of target words based on age groups. Thus, it seemed that the eye movements of the two age groups might be affected by frequency/predictability factors in slightly different ways.

The visual and linguistic demands of written Chinese are very different from those of alphabetic texts. Even so, researchers have observed a similar difference in eye movement behaviors between young and older readers of Chinese; that is, studies have consistently revealed that older Chinese readers fixate on target words for a longer time than their younger counterparts (Zang et al. 2016). Wang and her collaborators replicated larger frequency and predictability effects on fixation time measures for older readers of Chinese when they read twocharacter-words than for their young counterparts (Wang et al., 2018a, 2018b; Zhao et al., 2019). However, there were still distinctions based on impact of age on eye movement control between readers of Chinese and alphabetic languages; this implies that older English adult readers demonstrate longer forward eye movements, and skip words more often than their younger counterparts (see, Rayner et al., 2006, 2009). Although, recent evidence has revealed that there is no difference between the two age groups in terms of skipping rate and saccade length (Choi et al., 2017). Growing evidence on the topic of Chinese reading has indicated that older readers make shorter forward saccades and skip words more infrequently than young adult readers (Wang et al., 2018a, 2018b; Zang et al., 2016; Li et al., 2018). Thus, it seems that older Chinese readers employ a more careful strategy for eye movements, as compared to older readers of alphabetic texts. Therefore, based on the findings of the aforementioned studies, it is explicit that the effect of age on eye movement behavior is both language specific and universal.

As an important aspect of eye movement control during reading, saccade targeting to word seems to differ across Chinese and alphabetic languages. For most alphabetic writing systems, readers project their eyes to a specific location of a word, which is called preferred-viewing location (PVL; Rayner, 1979). It has been confirmed that linguistic characteristics of a word, such as predictability, did not impact the initial landing position of the eyes for target words (Rayner, Binder, Ashby, \& Pollatsek, 2001). In contrast, previous studies failed to find reliable evidences that Chinese readers target saccade to PVL during reading. However, evidence has shown that a target word can easily be processed, in that their frequency and predictability modulate the lengths of saccades targeting. Specifically, Liu and his collaborators have confirmed that target-word frequency modulates the lengths of incoming and outgoing saccades for target words, with a shorter saccade to/from low-frequency word (Liu, Reichle, \& Li, 2015, 
80

81

82

83

84

85

86

87

88

89

90

91

92

93

94

95

96

97

98

99

100

101

102

103

104

105

106

107

108

109

110

111

112

113

114

115

116

117

118

119

2016; Liu, Huang, Li, \& Gao, 2017); when compared with less predictable target words, highly predictable target words elicited longer saccades (Liu, Guo, Yu, \& Reichle, 2018). According to their findings, saccade amplitude is adjusted dynamically using lexical processing. Readers lengthen an impending saccade if the processing of the parafoveal or foveal word is easy (Liu et al., 2015; Liu, Huang,Gao, \& Reichle, 2017).

It is known that older Chinese readers adopt a more careful saccade strategy during reading, which may be owing to their poorer word processing, possibly due to a visual decline with age and/or additional visual requirements for the Chinese text (Owsley, 2011; Zhang et al., 2012; Wang et al., 2018a, 2018b; Zang et al., 2016; Li et al., 2018). Previous studies have revealed that word length produced a similar landing position, probability, and duration of eye movement in both young and older adult readers of English(Rayner et al., 2006; Paterson et al., 2015). von der Malsburg, Kliegl, and Vasishth (2014) also observed an insignificant interaction effect of aging with word length on scan-path. Whereas, a Chinese study recently revealed a lager word length effect on older Chinese readers than their young counterparts both in terms of fixation time and saccade length(Li et al., 2018). As has been noted earlier, growing evidence on how age impacts Chinese reading has consistently shown that older readers yield larger frequency and predictability effects on fixation time (i.e., gaze duration and total reading time). However, little is known about whether or not this impact of age could be categorized into saccades behaviors (i.e., length of impeding incoming/outgoing saccades for target words). Do these two effects of linguistic characteristics on saccades length change with age in Chinese readers? Exploring this would be undeniably helpful to reveal the factors responsible for the effect of age on eye guidance in Chinese readers. In the present study, we focus on how age mediates the effects of word frequency and predictability on the lengths of impending incoming/outgoing saccades for a target word.

How long of the impending saccade is related to parafoveal and foveal processing (Liu et al., 2015, 2018). Despite research conducted by Liu and his collaborators investigating the individual effects of word predictability and frequency on the length of impending saccades among a group of young adults (Liu et al., 2015, 2016, 2017), questions regarding how these two factors contribute, additionally or interactively, to saccade length, and whether or not age impacts these effects, still persist. Prior literature has suggested the need to predict differences in basic eye movement in terms of age; currently, it suggests that older adults need more time, fixate longer, and exhibit short saccade lengths during sentence comprehension as compared to young adults. However, we have mainly focused on the different effects of frequency/predictability or their interaction on the length of impending saccades for old adult readers. Encouraged by previous studies, we manipulated predictability and frequency orthogonally. The impact of age on lexical processing produced larger effects of frequency/predictability or their interactions on the fixation time of older readers. As the focus of present study, it predicates reliable interaction effects of age with two linguistic characteristics of words (i.e., frequency \& predictability) and their interaction on the length of impending saccades. 
120 Methods

121 Ethical consideration

122 The Center for Cognition and Brain Disorders of Hangzhou Normal University granted 123 ethical approval to carry out the study within its facilities (Approval Number, 20190408). Data 124 were anonymously collected after participants provided written informed consent by signing a 125 form prior to their participation.

126

127

128

129

130

131

132

133

134

135

136

137

138

139

140

141

142

143

144

145

146

147

148

149

150

151

152

153

154

155

156

157

158

159

\section{Participants}

Eighty young adults from Shanxi Normal University, and 40 older adults participated in the experiments. Older adults were recruited from the local community and included retired university teaching staff. All older adult participants were aged over 60 years $(M=62.40$, $\mathrm{SD}=2.01$ ). Young adults included students from Shanxi Normal University. These two groups did not differ in number of years of schooling (young adults: $\mathrm{M}=13.38, \mathrm{SD}=0.49$; old adults: $\mathrm{M}=13.43, \mathrm{SD}=1.96, \mathrm{t}=-0.216, \mathrm{p}>0.05)$. All participants were right-handed, had normal or corrected-to-normal vision; there was no difference between the two age groups in terms of corrected vision which was measured using the Tumbling E acuity chart (older: $M=4.98$, $S E=0.121$; younger: $M=4.99, S E=0.08 ; t=0.269, p>0.05$ ). All of them were native Chinese speakers. After completing the reading tasks, they were paid $¥ 30$ for participation.

\section{Apparatus}

An Eye Link II device, which was manufactured by SR Research Ltd., was used to record eye movements of the participants. It is a kind of infrared video-based tracking system. The camera of this device samples at a rate of $500 \mathrm{~Hz}$. The sentence stimuli were presented in black against a white background. Participants sat $45 \mathrm{~cm}$ away from the monitors, which were 19-inch DELL LCD devices with a refresh rate of $60 \mathrm{~Hz}$ and $1024 \times 768$ pixel resolution. The sentences were displayed in Song font, with each Chinese character subtending approximately a 1.32 degree visual angle.

\section{Design and stimuli}

The experiment followed a 2 (frequency of target words: high vs low) $\times 2$ (predictability of target words: predictable vs unpredictable) $\times 2$ (group: young adults vs old adults) design. Participants read 40 framed sentences which contained the target words. Example of these sentences are shown in Table 1. All the target words were composed of two characters, in which half of the these were high-frequency words, and another half were low-frequency words. Word and character frequencies were calculated using occurrences per million characters as a standardized measure, based on the database of Modern Chinese corpus word frequency and the database of Modern Chinese corpus character frequency, respectively, which were available at http://corpus.zhonghuayuwen.org/. The cut-off for high-frequency target words was more than 50 occurrences per million characters, while that for low-frequency words was less than 5 occurrences per million characters. As seen in Table 2, the mean frequency of words in the high- 
160 frequency condition is about 30 times that of the mean frequency in the low-frequency condition.

161

162

163

164

165

166

167

168

169

170

171

172

173

174

175

176

177

178

179

180

181

182

183

184

185

186

187

188

189

190

191

192

193

194

195

196

197

198

199

Thus, the word frequency manipulations in the current study were wider than those in previous studies (Wang et al., 2018a, 2018b). In addition, half of the target words were predictable from prior context, while half were unpredictable from the prior context. A group of 19 participants who did not participate in the experiment were asked to assess the predictability of the target words. They were shown the sentence frame up to, but not including, the target word and asked to complete the sentence. It was found that predictable target words were generated $74 \%$ of the time, whereas unpredictable target words were generated less than $1 \%$ of the time.

\section{Table 1 insert here}

\section{Table 2 insert here}

All four word types were balanced in terms of character frequency and strokes ( $p s>0.05)$, as seen in Table 2. There were no differences in word predictability, between predictable high frequency target words (HF-P) and predictable low frequency target words (LF-P), and between unpredictable low frequency target words (LF-U) and unpredictable high frequency target words (HF-U; $p s>0.05$ ), nor were there differences in word frequency between HF-P and HF-U, and between LF-U and LF-P. As seen in Tables 1 and 2, we developed two kinds of framed sentences. The first contained HF-P and LF-U target words, and the second contained LF-P and HF-U target words. It should be noted that words prior to the target word were also twocharacters in length, and were also balanced in terms of word frequency, character frequency, and strokes. The characters of pro-target words are shown in Table 3. There was no difference between the two kinds of prior target words in terms of word/character frequency and strokes $(p s>0.05)$. Fillers were not used in the present study.

\section{Procedure}

Table 3 insert here

When participants arrived, they were instructed to read sentences silently to understand their meaning and respond manually to comprehension questions. Then, experiment began with a 3horizontal-point calibration followed by 12 practice trials and 40 experimental sentences. All 40 experimental sentences were sampled using a Latin square to ensure that these sentences were shown in equal frequency in the 4 conditions. These 40 experimental sentences were displayed in a randomized order. The sentences were replaced during $28.6 \%$ of trials after a comprehension question with a YES or NO response (a total of 16 comprehension questions, with equal numbers of YES and NO answers). The eye tracker was engaged for a new calibration prior to each trial to ensure accuracy of eye tracing data if necessary; that is, we would conduct a re-calibration if error from drift correction of the present trial was greater than $0.5^{\circ}$. The entire experiment was completed in less than half an hour.

\section{Data analysis}

Comprehension accuracy was lower for older adults than their younger counterparts $(94.5 \%$ vs $\% 77.3 \%, p<0.001)$. This may be partly owing to older adults having difficulty with manual responses, even when they know the right answers (Wang et al., 2018b). We computed the 
200 correlations between older adults' comprehension accuracy, with their reading time for 201 sentences, and global measures of their eye movements (all $r s<0.25$, all $p s>0.05$ ). Therefore, 202 these findings indicated that the variations of older readers' comprehension scores did not 203 produce qualitative differences in reading performance or eye movement behavior, and there was 204 no evidence that indicated differences in reading strategy between high- and low-scoring older 205 readers. Eye movements at sentence-level and word-level were analyzed using the R Statistics 206 Package. Continuous data were analyzed using the LMM, and binary variables were analyzed 207 using GLMM. Complicated models including random slopes posed a problem of convergence; 208 therefore, we used maximal random effect structures, as suggested by Barr, Levy, Scheepers, and 209 Tily (2013), with participants and stimuli as crossed random effects. Age group was the only 210 fixed factor for the sentence-level measures analysis. Age group, frequency, predictability, and 211 their interactions were treated as fixed factors for word-level measures analysis(coded as sum 212 contrasts $-1 / 2$ vs $1 / 2$ for young and old adults, predictable vs unpredictable, and for high and low 213 frequency). Contrasts in the main effects were defined using sliding contrasts in the MASS 214 package (Venables \& Ripley, 2002). Effects based on both raw and $\log 10$-transformation were 215 reported for continuous variables. Regression coefficients $(b)$, standard errors $(S E), t(t=b / S E)$, 216 and $p$ values were reported. Models were fitted with the lme4 package (ver. 1.1-19; Bates, 217 Mäechler, Bolker, \& Walker, 2015) and $p$-values were estimated with the lmerTest package (ver. 218 3.0-1) in R (ver. 3.5.2; R Development Core Team, 2016). When analyzing raw data of gaze 219 duration and log-transformed data of total reading time, no convergence was observed in the 220 maximal random effect structures model, and so the models used for analyzing these data only 221 included random effect of participants.

222 Eye movements at sentence-level and word-level were recorded. Sentence-level measures 223 224

225

226

227

228

229

230

231

232

233

234

235

236

237

238 239 included sentence reading time (SRT), average fixation duration (AFD; mean duration of all fixation while reading a sentence), fixation count (FC), average saccade length (ASA; mean length of all saccades), regression number (RegNO; backward saccades number), word-level measures comprising fixation (time/probability), and saccade measure (length) of target words. Fixation time measures for target words included first fixation duration (FFD; the duration of the first fixation on the word irrespective of the number of fixations), gaze duration (GD; the sum of all fixations' duration on the word before moving to another word), and total reading time (TRT; sum of all fixations' duration). Go-past time (Go-past) is the sum of fixation duration from when the current area of interest is first fixated upon until one's eyes enter an interest area with a higher id. FFD and GD were treated as measures of first-pass reading, and TRT was treated as a measure of second-pass reading because it includes regressive re-reading time. Go-past was treated as a measure of difficulty in integrating a target word with the context before moving on in a sentence. Fixation probability measures were associated with target words, and included the probability of skipping (Skip.pro) or re-fixating (Refix.pro) on a target word. Saccade measures comprised incoming saccade length (ISL; length of the first-pass progressive saccade resulting in fixation on the target word) and outgoing saccades length (OSL; length of the first-pass progressive saccade launched away from the target word). 
240

241

242

243

244

245

246

247

248

249

250

251

252

253

254

255

256

257

258

259

260

261

262

263

264

265

266

267

268

269

270

271

272

273

274

275

276

277

278

\section{Results}

\section{Sentence-level measures analyses}

The mean and standard errors of the sentence level measures are shown in Table 4, and corresponding statistical effects are summarized in Table 5. The results show that an older adult requires longer fixations and regressions to comprehend a sentence than their young counterparts. The pattern of these effects is consistent with the fact that older adults experience greater difficulty in reading (Wang et al., 2018a, 2018b; Zang et al., 2016). However, contrary to what we had expected, we did not observe any significant differences in the average saccade length between the two age groups. As previous studies on effects of age on Chinese readers have also shown no differences in age groups in terms of saccade length, the our findings regarding sentence-level measures were consistent with previous research (Zhao et al., 2019; Li et al., 2018).

\section{Word-level measures analyses}

Table 4 insert here

Table 5 insert here

Fixation time. A total of 4268 observations contributed to the analyses. As shown in Tables 6 and 7, differences were observed between the two age groups of readers in terms of fixation time measures. Specifically, older readers fixated on target words longer than young readers. Reliable effects of predictability were observed; specifically, predictable targets were fixated upon for a shorter time than unpredictable targets. A similar pattern was observed in the effect of frequency also, wherein frequency had a significant impact on FFD, GD, Go-past, and TRT. The present observations on the interaction effects are also important in the present study. Reliable interaction effect of Predictability $\times$ Frequency on raw GD was due to the larger frequency effect of unpredictability as compared to predictability ( $39 \mathrm{~ms}$ vs $22 \mathrm{~ms}$ ). Reliable interaction effects of Group $\times$ Predictability and Group $\times$ Frequency on raw TRT were due to consistently large frequency and predictability effects on older adult readers than on young adult readers (group differences of frequency effect: $33 \mathrm{~ms} v s 75 \mathrm{~ms}$; group differences of predictability effect: 117 ms vs $163 \mathrm{~ms}$ ). Reliable interaction effects of Group $\times$ Frequency on both raw and logtransformed GD as well as go-past were due to the larger frequency effects on older readers as compared to younger readers (frequency effects for older: $\mathrm{GD}=53 \mathrm{~ms}$; go-past $=76 \mathrm{~ms}$; frequency effects for young adult readers: $\mathrm{GD}=17 \mathrm{~ms}$; go-past $=17 \mathrm{~ms}$ )

\section{Table 6 insert here}

Table 7 insert here

Probability measures. A total of 4800 observations contributed to the analyses. As shown in Tables 6 and 8, the two age groups of readers significantly differed in the probability of skipping and re-fixating on target words. Older readers skipped target words less often, and refixated on them more than younger readers. Significant predictability effects were found on Skip.Pro and Refix.Pro, with a higher skip and less re-fixation probability for predictable as 
279 compared to unpredictable words. Frequency effect was reliable only on Refix.pro. No 280 interaction effects were reliable on any probability measures.

281 Saccade length. A total of 3908 and 3441 observations contributed to ISL and OSL 282 analyses, respectively. As shown in Tables 6 and 8, analyses revealed no significant effects of 283 group, frequency, and predictability on raw data of both ISL and OSL, but reliable frequency 284 effects on log-transformed OSL was observed. Reliable interaction effects of Group $\times$ 285 Predictability on ISL was due to a negative predictability effect on young adult readers $(-0.08$

287

288

289

290

291

292

293

294

295

296

297

298

299

300

301

302

303

304

305

306

307

308

309

310

311

312

313

314

315

316 char), but a reliable positive effect on older readers ( 0.14 char). As shown in Table 8 , a threeway interaction (Group $\times$ Predictability $\times$ Frequency) in log-transformed ISL was observed. We employed models for analyzing the data of log-transformed ISL for younger and older readers separately to clarify this interaction, and observed opposite values for these two groups, although both interactions of Predictability $\times$ Frequency were not reliable (young readers: $b=-0.054$, $S E=0.039, t=-1.391, p=0.172$; maximal random effect structures model did not converge for older readers, so this model on only included the random effect of participants: $b=0.013$, $S E=0.017, t=0.748, p=0.454)$. As for OSL, reliable interaction effects of Group $\times$ Frequency was also due to a negative effect of frequency on the young readers $(-0.06$ char) and a positive effect on older readers (0.08 char). To further investigate the three-way interaction (Group $\times$ Predictability $\times$ Frequency) in OSL, we employed models for analyzing the data of younger and older readers separately, which also yielded opposite interaction values of Predictability $\times$ Frequency for these two groups (results of raw OSL for young readers: $b=-0.026, S E=0.157, t=-$ $0.168, p=0.867$; results of raw OSL for older readers: $b=0.234, S E=0.185, t=1.265, p=0.213$; results of log-transformed OSL for young readers: $b=-0.010, S E=0.035, t=-0.287, p=0.776$; results of log-transformed OSL for older readers: $b=0.052, S E=0.024, t=2.168, p=0.036$ ), all of these interactions were not reliable.

\section{Discussion}

The present study assessed how the effects of frequency and predictability, and their interaction in eye movement behavior, especially in case of saccades, on Chinese readers were mediated by age. This study contributes to the growing evidence on the impact of age on word processing in several ways. First, the results of the word-level measures analyses replicated those of previous studies, which state that older readers need more fixation time for comprehending text. However, we could not determine the effect of age on average saccade length, which is also consistent with the scope of the findings of a recent study by Zhao et al (2019). Second, with regards to the fixation time measures on target words, older readers fixated on these words for a longer time and re-fixated on them more often than younger readers, which also mirrors previous findings of studies on both Chinese and alphabetic text reading (Kliegl et al., 2004; Rayner et al., 2006, 2009, 2010; Kemper \& McDowd, 2006; Kemper \& Liu, 2007; Paterson et al., 2013a, 2013b, 2013c; McGowan et al., 2014; Zang et al., 2016; Wang et al., 2018a, 2018b). Third, we 
317 replicated age-related differences in skip measure; specifically, older Chinese readers skipped 318 target words less often than young adult readers (Zang et al., 2016; Wang et al., 2018a, 2018b).

319 Moreover, we observed the reliable impact of age on frequency and predictability, and their 320 interaction effects on certain eye movement measures, which notably reveal eye movement

321

322

323

324

325

326

327

328

329

330

331

332

333

334

335

336

337

338

339

340

341

342

343

344

345

346

347

348

349

350

351

352

353

354

355 control and word processing in Chinese reading.

For the interaction effects involving age with the linguistic characteristics of predictability and frequency, our findings further suggest that age promotes the effects of frequency. Previous studies have revealed larger frequency effects on fixation time measures, such as first fixation duration, gaze duration, and total reading time, in alphabetic text reading (Kliegl et al., 2004; Rayner et al., 2006). Conversely, Wang and her collaborators failed to report any larger frequency effects on older Chinese readers when analyzing log-transformed fixation time; however, they reported larger frequency effect on raw fixation time measures, such as gaze duration and total reading time, which was owing to older readers demonstrating very long fixation durations for low frequency words (Wang, et al.,2018a; 2018b). The interaction effect between age group and frequency is extremely difficult to examine. Analyses on log-transformed data are likely to distort the ratio scale properties, which can lead to effects impacted by distribution tail to go unnoticed (Wagenmakers, Krypotos, Criss, \& Iverson, 2012; Lo, \& Andrews, 2015); therefore, as compared to log-transformed fixation data, analyzing raw data is more convenient for detecting such interactions. However, we observed a larger frequency effect on log-transformed gaze duration and go-past time for older Chinese readers, which may partially be owing to a much more potent manipulation of word frequency.

Larger predictability effects on older Chinese readers were observed in fixation time measures, which suggest that older readers make greater use of context information for word identification than their young counterparts (Zhao, et al., 2019). Our findings also help understand the role of age in utilizing context cues to promote eye movement and word processing during reading. We could not determine a reliable interaction effect of age and predictability on fixation measures in the first fixation duration and go-past time, but marginal or reliable interaction effects on raw data of gaze duration and total reading were observed. Interaction effects involving older groups with other variables are not easy to examine, but raw data help in detecting such interactions. Therefore, our findings have contributed, at least partly, to the evidence that older readers make greater use of context to identify words during reading (Choi et al., 2017; Zhao et al., 2019). Our results, of course, also further elaborate on how predictability impacts word identification in its interaction with frequency. Despite many observations by studies on eye tracking regarding the contributions of predictability and frequency to fixation durations while reading alphabetic text (Kennedy, Pynte, Murray, \& Paul, 2013; Rayner, Ashby, Pollatsek, \& Reichle, 2004), questions regarding whether these two factors contribute additionally, or interactively, to eye movement control in Chinese reading, and whether age impacts their interactions, still persist. Our results demonstrate the additional contributions of predictability and frequency on all measures of fixation for both age groups. 
356 Thus, our findings suggest that, at least for older readers, interaction effects of frequency and

357

358

359

360

361

362

363

364

365

366

367

368

369

370

371

372

373

374

375

376

377

378

379

380

381

382

383

384

385

386

387

388

389

390

391

392

393

394

395

predictability on fixation time appears to be similar across scripts and age groups.

The probability of skipping and re-fixating on target words revealed some differences between Chinese and alphabetic text. It was found that word skipping rate was insensitive to the interactions of age and frequency and that of age and predictability for Chinese readers, which is a finding consistent with that of previous studies (Wang, et al.,2018a; Zhao et al., 2019). However, this finding is inconsistent with that of studies which focused on reading of alphabetic text. Specifically, in English reading, age also promotes the frequency effect on skipping rate of the target word, but does not interact with predictability (Rayner et al., 2006); however, in German reading, it was found that high predictability increased young adult readers' probability of skipping target words (Kliegl et al., 2004). We also observed no significant interactions between aging, frequency, and predictability for re-fixating rate of target words. This null finding was only partly consistent with the findings of studies on reading of alphabetic text. In English reading, there is no indication that older readers' re-fixating rate of words might be more likely to be impacted by frequency and predictability. However, in German reading, Kliegl et al. (2004) found that highly predictable words decreased older readers' probability of multiple fixations on target words. The results of the present study showed that the effects of frequency and predictability on skipping of and re-fixating rate of target words were generally insensitive to age. Thus, it demonstrates a different pattern of the effects of age on skipping and re-fixating across Chinese and alphabetic text reading.

Perhaps the most important contribution of the present study is that it helps understand how text processing affects saccades targeting. We suppose that the reason we could not determine reliable effects of frequency and predictability, and their interaction, on both the length of incoming/outgoing saccades (although reliable effects of frequency and predictability were observed on log-transformed data in terms of length of outgoing saccades, with a shorter outgoing saccade when predictability or frequency was low), is that each Chinese character subtended a larger visual angle than that used in the study by Liu and his colleagues $\left(1.32^{\circ} \mathrm{vs} 1^{\circ}\right.$, Liu et al., 2015, 2016, 2017, 2018), which may eliminate some effects of parafoveal word processing. Nevertheless, for both raw and log-transformed length measures, a reliable interaction effect of age and predictability on incoming saccade length was observed. Specifically, older readers yielded a different predictability effect on incoming saccade length. Previous research found that young Chinese adult readers could use predictability information for promoting the processing of parafoveal characters of the target word (Su, Liu, \& Cao, 2016). The predictability of words presumably facilitates the preprocessing of these words (Balota, Pollatsek, \& Rayner, 1985; White, Rayner, \& Liversedge, 2005a; Schotter, Lee, Reiderman, \& Rayner, 2015), and the interaction effect of age and predictability on incoming saccade length suggests that older readers either experience a decline in the preprocessing of low predictability target words or they make greater use of context to identify words.

Reliable interaction effects of age and frequency on fixation time (i.e. gaze duration, go-past time, and total reading time) indicated that older people were more susceptible to foveal load

Peer) reviewing PDF | (2019:08:40725:3:0:NEW 6 Feb 2020) 
396

397

398

399

400

401

402

403

404

405

406

407

408

409

410

411

412

413

414

415

416

417

418

419

420

421

422

423

424

425

426

427

428

429

430

431

432

433

434

manipulated by word frequency (see Henderson \& Ferreira, 1990; Henderson \& Ferreira, 1990; Kennison \& Clifton, 1995; Kliegl, Nuthmann, \& Engbert, 2006; White, Rayner, \& Liversedge, 2005b; Kliegl, 2007, for word frequency manipulating foveal load). Corresponding to this, reliable interaction effects of age and frequency on outgoing saccades length was observed. We found a negative frequency effect on outgoing saccades length for older readers, which may be a result of their longer fixation on the infrequency of target words. It should be noted that foveal load affects information that can be extracted from the parafoveal; moreover, word frequency modulated outgoing saccades length also reflect the functional role of parafoveal processing in determining saccade targeting (Liu et al., 2015). Thus, the reliable interaction effects of age and frequency on outgoing saccades length also suggest a decline of word processing in determining eye movement during reading. This discrepancy observed in both older and young adult readers when considering the effect of predictability on incoming saccade length and effects of frequency on outgoing saccades length may suggest decline in word processing of older Chinese readers. Future studies must investigate why age impacts the effects of frequency on outgoing saccades length, which is a measure of relatively later word processing, rather than on incoming saccade length. Our findings suggest that word identification in Chinese text depends on readers first identifying characters that might combine to form a word (Zang et al., 2011; Ma, Li, \& Rayner, 2015); thus, frequency effects of two-character words may emerge slowly in Chinese reading.

Taken together, the interaction of age with frequency and predictability affect the amplitude of saccades, which is an important finding as it suggests that age impacts word processing of, possibly both, in parafoveal and foveal vision. This study particularly aimed to assess the impact of age on interactions involving word frequency and predictability in Chinese reading. The absence of interaction effects between word frequency and predictability on eye movements measures in young adult readers indicated that word frequency and predictability impacted a different stage of word processing; this finding is consistent with previous findings of studies on reading alphabetic text (Ashby, Rayner, \& Clifton, 2005; Gollan, Slattery, Goldenberg, Van Assche, Duyck, \& Rayner, 2011; Hand, Miellet, O’Donnell, \& Sereno, 2010; Slattery, Staub, \& Rayner, 2012). Conversely, the reliable interaction effect of the three variables on outgoing saccades length produced an interaction of frequency and predictability that was positive for older readers and negative for young adult readers (notably, the reliable effects of a three-way interaction, namely Predictability $\times$ Frequency $\times$ Group, on both raw and log-transformed outgoing saccades length were observed). Both parafoveal and foveal lexical processing difficulty is used to determine how far one's eyes would move during reading (Liu et al., 2015, 2016, 2017, 2018). Significant interaction effects of Group $\times$ Predictability on incoming saccade length suggests a decline in parafoveal lexical processing, while reliable interaction effects of Group $\times$ Frequency on outgoing saccades length suggests a decline in foveal lexical processing for older readers. Therefore, this three-away interaction effect on outgoing saccades length may result from a decline in both parafoveal and foveal lexical processing for old readers. 
435

436

437

438

439

440

441

442

443

444

445

446

447

448

449

450

451

452

453

454

455

456

457

458

459

460

461

462

463

464

465

466

467

468

469

470

471

472

473

474

Several limitations of the present study must be acknowledged. First, the eye-tracker we used has a relatively low spatial and temporal resolution than others. Although this may not be a fatal defect, upon comparison with the methods used in previous studies, we believe that this needs be acknowledged. Second, comprehension scores were lower for older adult readers. It is unlikely that older adult readers did not comprehend sentences because they were all retired teaching staff from the university; they were not very old. Unfamiliarity with computer equipment could have also affected their manual responses. The final limitation is related to validity of the findings. The launch site of impeding saccade may systematically vary across young and older adults, results of saccade length are also likely be systematically affected by higher re-fixation rates of the older adults. But we focused on the interactions of predictability and frequency with age on these two kind of impeding saccade length, the results of reliable interactions must be, at least partly, resulting from these systematical differences. We tested our hypothesis multiple times, and came to the conclusion that using Bonferroni corrections was the most effective method to validate our results (von der Malsburg \& Angele, 2016). Considering our hypothesis that age interacts with linguistic characteristics based on saccade length in Chinese reading, we observed three interactions for each of the two saccade length measures; therefore an alpha of 0.0083 is appropriate in this context (new $\alpha=0.05 / 6 \approx 0.0083$ ). Barring the three-away interaction effects on log-transformed outgoing saccades length, and Group $\times$ Predictability interaction effect on raw data and incoming saccade length, we have interpreted various null results of interactions in our discussion. Although the extent to which extent statistic power has been reduced by Bonferroni corrections is unclear, a reliable three-way interaction may be owing to the two interactions of age with frequency and with predictability. Discussing these "null" results have has helped clarify the interactions effects of age on impeding saccade length.

The present study, and other similar studies, may contribute to a better understanding of language specific characters and how reading behaviors change with age, specifically in readers of the Chinese text. In summary, we found that effects related to age, word frequency, and predictability appear to be similar across alphabetic and non-alphabetic text, when assessed with measures of fixation time. Measures of saccade length maybe the most informative for understanding how age impacts word processing in Chinese readers. Specifically, a unique pattern of results was observed in terms of measures of incoming and outgoing saccades length. Thus, present study provides evidence suggesting that these age-related effects differ qualitatively across alphabetic text reading. Previous research has already elaborated on this qualitative difference. Paterson et al. (2015) found that young and older adult readers of alphabetic text demonstrated similar patterns of landing position on both short and long words. This finding suggests that eye guidance is preserved in older adult readers during alphabetic text reading. We suppose that this was due to differences in the behaviors of alphabetic text and Chinese text readers. Specifically, reading alphabetic text requires the use of visual cues for guidance on where to move one's eyes, and requires the reader to focus on a target word regardless of word length (Rayner, 1998, 2009). Whereas, reading Chinese text requires eye movements, that is saccade targeting, to be modulated by the parafoveal and foveal lexical 
475 processing (Liu et al., 2015, 2016, 2017). Our findings demonstrate that age interacts with lexical 476 processing to effect saccade length. Ultimately, observing effects of age on eye movements 477 while reading in Chinese may help in further understanding of the characteristics that underlie 478 changes in reading behaviors over time.

\section{Conclusions}

480 Results of present study suggest that the word processing function of older Chinese readers in terms of saccade targeting declines with age.

482

\section{Acknowledgements}

This research was supported by Zhejiang Provincial Natural Science Foundation of China under Grant No.LY18C090002, and also supported by Foundations from Hangzhou Normal University (No. 4045C5021820444, 4045C51918008816). There was no additional funding received for this study. We thanks Titus von der Malsburg for providing analyzing code.

\section{References}

Ashby, J., Rayner, K, \& Clifton, C.(2005). Eye movements of highly skilled and average readers: Differential effects of frequency and predictability. Quarterly Journal of Experimental Psychology. 58, 1065-1086.

Balota, D. A., Pollatsek, A., \& Rayner, K. (1985). The interaction of contextual constraints and parafoveal visual information in reading. Cognitive Psychology, 17, 364-390.

Barr, D.J., Levy, R., Scheepers, C., \& Tily, H.J. (2013). Random effects structure for confirmatory hypothesis testing: keep it maximal. Journal of Memory and Language, 68, 255-278.

Bates, D., Mächler, M., Bolker, B., \& Walker, S. (2015). Fitting linear mixed-effects models using lme4. Journal of Statistical Software, 67, 1-48.

Choi, W., Lowder, M. W., Ferreira, F., Swaab, T. Y., \& Henderson, J. M. (2017). Effects of word predictability and preview lexicality on eye movements during reading: A comparison between young and older adults. Psychology and Aging, 32, 232242.

Gollan, T., Slattery, T. J., Goldberg, D., van Assche, E., Duyck, W., \& Rayner, K. (2011). Frequency drives lexical access in reading but not in speaking: The frequency-lag hypothesis. Journal of Experimental Psychology: General, 140, $186-209$.

Hand, C. J., Miellet, S., O’Donnell, P. J., \& Sereno, S. C. (2010). The frequency-predictability interaction in reading: It depends on where you're coming from. Journal of Experimental Psychology: Human Perception and Performance, 36, $1294-1313$.

Henderson, J. M., \& Ferreira, F. (1990). Effects of foveal processing difficulty on the perceptual span in reading: Implications for attention and eye movement control. Journal of Experimental Psychology: Learning, Memory, and Cognition, 16, 417-429

Kemper, . S. , \& McDowd, J. (2006). Eye movements of young and older adults while reading with distraction. Psychology and Aging, 21, 32-39.

Kemper, S., \& Liu, C. J. (2007). Eye movements of young and older adults during reading. Psychology and Aging, 22, 84-94.

Kemper, S., Crow, A., \& Kemtes, K. (2004). Eye-fixation patterns of highand low-span young and older adults: Down the garden path and back. Psychology and Aging, 19, 157-170.

Kennedy, A., Pynte, J., Murray, W. S., \& Paul, S.A. (2013). Frequency and predictability effects in the Dundee Corpus: An eye movement analysis. The Quarterly Journal of Experimental Psychology, 66, 601-618.

Kennison, S. M., \& Clifton, C., Jr. (1995). Determinants of parafoveal preview benefit in high and low working memory capacity readers: Implications for eye movement control. Journal of Experimental Psychology: Learning, Memory, and Cognition, 21, 68-81.

Kliegl, R. (2007). Towards a perceptual-span theory of distributed processing in reading: A reply to Rayner, Pollatsek, Drieghe, Slattery, and Reichle (2007). Journal of Experimental Psychology: General, 136, 530-537.

Kliegl, R., Grabner, E., Rolfs, M., \& Engbert, R. (2004). Length, frequency, and predictability effects of words on eye movements in reading. European Journal of Cognitive Psychology, 16,262-284.

Kliegl, R., Nuthmann, A., \& Engbert, R. (2006). Tracking the mind during reading: The influence of past, present, and future words on fixation durations. Journal of Experimental Psychology: General, 135, 12-35.

Laubrock, J., Kliegl, R., \& Engbert, R. (2006). SWIFT explorations of age differences in eye movements during reading. Neuroscience and Biobehavioral Reviews, 30, 872-884.

Li, S., Li., Wang, J. McGowan, V. A., \& Paterson, K.B. (2018). Effects of word length on eye guidance differ for young and older Chinese readers. Psychology and Aging, 33, 685-692. 
Liu, Y., Huang, R., Gao, D., \& Reichle, E. D. (2017). Further tests of a dynamic-adjustment account of saccade targeting during the reading of Chinese. Cognitive Science, 41, 1264-1287.

Liu, Y., Huang, R., Li, Y., \& Gao, D. (2017). The Word Frequency Effect on Saccade Targeting during Chinese Reading: Evidence from a Survival Analysis of Saccade Length. Frontiers in psychology, 8, 116.

Liu, Y. , Guo, S. , Yu, L. , \& Reichle, E. D. . (2018). Word predictability affects saccade length in chinese reading: an evaluation of the dynamic-adjustment model. Psychonomic Bulletin \& Review. 25, 1891-1899

Liu, Y., Reichle, E. D., \&Li, X. (2015). Parafoveal processing affects outgoing saccade length during the reading of Chinese. Journal of Experimental Psychology: Learning, Memory, and Cognition, 41(4), 1229-1236.

Liu, Y., Reichle, E. D., \& Li, X. (2016). The effect of word frequency and parafoveal preview on saccade lengthduring the reading of Chinese. Journal of Experimental Psychology: Human Perception and Performance, 42(7), 1008-1025.

Lo, S., \& Andrew, S. (2015). To transform or not to transform: using generalized linear mixed models to analyse reaction time data. Frontier in Psychology. 6:1171.

Ma, G., Li, X., \& Rayner, K. (2015). Readers extract character frequency information from nonfixated-target word at long pretarget fixations during Chinese reading. Journal of Experimental Psychology: Human Perception and Performance, 41,1409-1419.

McGowan, V.A., White, S.J., Jordan, T.R., \& Paterson, K.B. (2014). Aging and the use of interword spaces during reading: Evidence from eye movements. Psychonomic Bulletin \& Review, 21, 740- 747.

Owsley, C. (2011). Aging and vision. Vision Research, 51, 1610-1622.

Paterson, K. B., Almabruk, A. A. A., McGowan, V. A., White, S. J., \& Jordan, T. R. (2015). Effects of word length on eye movement control: The evidence from Arabic. Psychonomic Bulletin \& Review, 22, 1443-1450.

Paterson, K.B., McGowan, V.A., \& Jordan, T.R. (2013a). Filtered text reveals adult age differences in reading: Evidence from eye movements. Psychology and Aging, 28, 352-364.

Paterson, K.B., McGowan, V.A., \& Jordan, T.R. (2013b). Effects of adult aging on reading filtered text: Evidence from eye movements. PeerJ 1:e63.

Paterson, K.B., McGowan, V.A., \& Jordan, T.R. (2013c). Aging and the control of binocular fixations during reading. Psychology and Aging, 28, 789-795.

R Development Core Team (2016). R: A language and environment for statistical computing. R foundation for statistical computing, Vienna, Austria. <http://www.R-project.org $>$.

Rayner, K. (1979). Eye guidance in reading: Fixation locations within words. Perception, 8, 21-30

Rayner, K. (1998). Eye movements in reading and information processing: 20 years of research. Psychological Bulletin, 124, $372-422$.

Rayner, K. (2009). Eye movements in reading: Models and data. Journal of Eye Movement Research, 2, 1-10.

Rayner, K., Ashby, J., Pollatsek, A., \& Reichle, E. (2004). The effects of frequency and predictability on eye fixations in reading: Implications for the E-Z Reader model. Journal of Experimental Psychology: Human Perception and Performance, 30, 720732

Rayner, K., Binder, K. S, Ashby, J., \& Pollatsek, A. (2001). Eye movement control in reading: Word predictability has little influence on initial landing positions in words. Vision Research, 41, 943-954.

Rayner, K., Castelhano, M. S., \& Yang, J. (2009). Eye movements and the perceptual span in older and younger readers. Psychology and Aging, 24, 755-760.

Rayner, K., Castelhano, M. S., \& Yang, J. (2010). Preview benefit during eye fixations in reading for older and younger readers. Psychology and Aging, 25, 714-718.

Rayner, K., Reichle, E. D., Stroud, M. J., Williams, C. C., \& Pollatsek, A.(2006). The effects of word frequency, word predictability, and font difficulty on the eye movements of young and elderly readers. Psychology and Aging, 21, 448-465.

Schotter, E. R., Lee, M., Reiderman, M., \& Rayner, K. (2015). The effect of contextual constraint on parafoveal processing in reading. Journal of Memory and Language, 83, 118-139.

Slattery, T. J., Staub, A., \& Rayner, K. (2012). Saccade launch site as a predictor of fixation durations in reading: Comments on Hand, Miellet, O'Donnell, and Sereno (2010). Journal of Experimental Psychology: Human Perception and Performance, 38 , 251-261.

Steen-Baker, A. A., Ng, S., Payne, B. R., Anderson, C. J., Federmeier, K. D., \& Stine-Morrow, E. A. L. (2017). The effects of context on processing words during sentence reading among adults varying in age and literacy skill. Psychology and Aging, $32,460-472$.

Su, H., Liu, Z., \& Cao, L. (2016). The effects of word frequency and word predictability in preview and their implications for word segmentation in Chinese reading: Evidence from eye movements. Acta Psychologica Sinica, 48, 625-626. $\square$

von der Malsburg, T., Kliegl, R., \& Vasishth, A. (2015). Determinants of scanpath regularity in reading. Cognitive Science, 39, $1675-1703$.

von der Malsburg, T., \& Angele, B. (2016). False positives and other statistical errors in standard analyses of eye movements in reading. Journal of Memory and Language, 94, 119-133

Wagenmakers, E. J., Krypotos, A. M., Criss, A. H., \& Iverson, G. (2012). On the interpretation of removable interactions: A survey of the field 33 years after Loftus. Memory \& Cognition, 40, 145-160.

Wang, J., Li, L., Li, S., Xie, F., Chang, M., Paterson, K. B., White, S. J., \& McGowan, V. A. (2018a). Adult age differences in eye movements during reading: The evidence from Chinese. Journals of Gerontology: Psychological Sciences, 73, 584-593. 
587

Wang, J., Li, L., Li, S., Xie, F., Liversedge, S. P., \& Paterson, K. B. (2018b). Effects of Aging and Text Stimulus Quality on the Word Frequency Effect during Chinese Reading. Psychology and Aging, 33, 693-712.

White, S. J., Rayner, K., \& Liversedge, S. P. (2005a). The influence of parafoveal word length and contextual constraint on fixation durations and word skipping in reading. Psychonomic Bulletin \& Review, 12, 466-471.

White, S. J., Rayner, K., \& Liversedge, S. P. (2005b). Eye movements and the modulation of parafoveal processing by foveal processing difficulty: A reexamination. Psychonomic Bulletin \& Review, 12, 891-896

Whitford, V., \& Titone, D. (2017). The effects of word frequency and word predictability during first- and second-language paragraph reading in bilingual older and younger adults. Psychology and Aging, 32, 158-177

Zang, C., Zhang, M., Bai, X., Yan, G., Paterson, K. B., \& Liversedge, S. P. (2016). Effects of word frequency and visual complexity on eye movements of young and older Chinese readers. The Quarterly Journal of Experimental Psychology, 69, 1409-1425.

Zhao, S., Li, L., Chang, M., Xu, T., Zhang, K., Wang, J., \& Paterson, K.B. (2019). Older adults make greater use of word predictability in Chinese reading. Psychology and Aging, 34, 780-790.

Zhang, X. X., Fang, Z., Du, Y. C., Kong, L. Y., Zhang, Q., \& Xing, Q. (2012). Centro-parietal N200: An event-related potential component specific to Chinese visual word recognition. Chinese Science Bulletin, 57), 1516-1532. 
Table $\mathbf{1}$ (on next page)

Table 1 
1 Table 1. Example Sentences

\begin{tabular}{|l|l|}
\hline Conditions & Sentence \\
\hline HF-P & $\begin{array}{l}\text { 公司经理在提高产品质量方面花费了大量精力。 } \\
\text { In older to improving product quality, the company manager put a lot of effort }\end{array}$ \\
\hline LF-U & $\begin{array}{l}\text { 公司经理在提高产品名声方面花费了大量精力。 } \\
\text { In older to improving product fame, the company manager put a lot of effort }\end{array}$ \\
\hline LF-P & $\begin{array}{l}\text { 外星人经常驾驶飞船去往地球的各个角落。 } \\
\text { Aliens often drive spacecraft to all corners of the earth. }\end{array}$ \\
\hline HF-U & $\begin{array}{l}\text { 外星人经常驾驶汽车去往地球的各个角落。 } \\
\text { Aliens often drive car to all corners of the earth. }\end{array}$ \\
\hline
\end{tabular}

2 (Note. $\mathrm{HF}=$ high-frequency targets; $\mathrm{P}=$ predictable targets; $\mathrm{U}=$ unpredictable targets; $\mathrm{LF}=$ low-frequency targets) 
Table 2 (on next page)

Table 2 the characters of target words .doc 
1 Table 2 the characters of target words (predictable, frequency, and stroke)

\begin{tabular}{ccccccc}
\hline Conditions & $\begin{array}{c}\text { Word } \\
\text { predictable }\end{array}$ & $\begin{array}{c}\text { Word } \\
\text { frequency }\end{array}$ & $\begin{array}{c}\text { First } \\
\text { character } \\
\text { frequency }\end{array}$ & $\begin{array}{c}\text { Second } \\
\text { character } \\
\text { frequency }\end{array}$ & $\begin{array}{c}\text { First } \\
\text { character } \\
\text { strokes }\end{array}$ & $\begin{array}{c}\text { Second } \\
\text { character } \\
\text { strokes }\end{array}$ \\
\hline HF-P & $0.74(0.14)$ & $111.60(62.59)$ & $792.27(537.11)$ & $597.95(435.01)$ & $7.45(2.87)$ & $7.60(2.68)$ \\
LF-U & $0.01(0.01)$ & $3.42(0.72)$ & $798.27(1038.02)$ & $684.40(545.69)$ & $7.25(3.54)$ & $7.25(2.17)$ \\
LF-P & $0.74(0.17)$ & $3.69(1.30)$ & $630.24(818.67)$ & $554.74(571.21)$ & $7.60(2.60)$ & $7.65(2.52)$ \\
HF-U & $0.01(0.02)$ & $113.94(60.21)$ & $729.21(531.07)$ & $768.29(580.95)$ & $7.20(2.53)$ & $7.65(1.53)$ \\
\hline
\end{tabular}

2 (Note: The standard deviations are given in parentheses)

3 


\section{Table 3(on next page)}

Table 3 the frequency, and stroke characters of pro-target word 
1 Table 3 the frequency, and stroke characters of pro-target word

\begin{tabular}{cccccc}
\hline $\begin{array}{c}\text { Pro-target } \\
\text { word in frame }\end{array}$ & $\begin{array}{c}\text { Word } \\
\text { frequency }\end{array}$ & $\begin{array}{c}\text { First character } \\
\text { frequency }\end{array}$ & $\begin{array}{c}\text { Second character } \\
\text { frequency }\end{array}$ & $\begin{array}{c}\text { First character } \\
\text { strokes }\end{array}$ & $\begin{array}{c}\text { Second character } \\
\text { strokes }\end{array}$ \\
\hline $\begin{array}{c}\text { HF-P } \\
\text { LF-U }\end{array}$ & $75.15(84.06)$ & $818.81(671.05)$ & $1285.75(1021.67)$ & $7.40(1.85)$ & $7.30(2.81)$ \\
\hline $\begin{array}{l}\text { LF-P } \\
\text { HF-U }\end{array}$ & $65.58(72.83)$ & $649.24(698.92)$ & $1331.60(961.52)$ & $7.90(1.89)$ & $7.50(2.06)$ \\
\hline
\end{tabular}

2 (Note: The standard deviations are given in parentheses)

3 


\section{Table 4(on next page)}

Table 4 The mean and standard errors of the sentence level measures across age groups 
1 Table 4 The mean and standard errors of the sentence level measures across age groups

\begin{tabular}{cccccc}
\hline & SRT & AFD & FC & ASA & RegNo \\
\hline Young & $3558(165.6)$ & $226(3.3)$ & $13.5(0.56)$ & $2.16(0.06)$ & $3.6(0.18)$ \\
older & $5541(234.1)$ & $247(4.7)$ & $20.0(0.79)$ & $2.11(0.08)$ & $4.8(0.25)$ \\
\hline
\end{tabular}

2 (Note: Means and standard errors are computed across subjects' means. The standard errors are given in parentheses. SRT,

3 sentence reading time in millisecond; AFD, mean fixation duration in milliseconds; FC, fixation number; ASA, mean saccade

4 length in character; RegNO, number of regressions.)

5 


\section{Table 5 (on next page)}

Table 5 Linear mixed-effects model analyses on sentence level measures 
1 Table 5 Linear mixed-effects model analyses on sentence level measures

\begin{tabular}{|c|c|c|c|c|c|c|c|c|}
\hline & \multicolumn{4}{|c|}{ SRT } & \multicolumn{4}{|c|}{ SRT log-transformed } \\
\hline & $b$ & $S E$ & $t$ & $p$ & $b$ & $S E$ & $t$ & $p$ \\
\hline Inter & 4551.5 & 163.7 & 27.81 & $<0.001$ & 3.601 & 0.017 & 214.695 & $<0.001$ \\
\hline \multirow[t]{2}{*}{ Group } & -1987.1 & 286.7 & -6.93 & $<0.001$ & -0.201 & 0.030 & -6.785 & $<0.001$ \\
\hline & \multicolumn{4}{|c|}{ AFD } & \multicolumn{4}{|c|}{ AFD log-transformed } \\
\hline Inter & 236.296 & 2.916 & 81.041 & $<0.001$ & 2.367 & 0.005 & 454.054 & $<0.001$ \\
\hline \multirow[t]{2}{*}{ Group } & -20.778 & 5.704 & -3.642 & $<0.001$ & -0.041 & 0.010 & -4.027 & $<0.001$ \\
\hline & \multicolumn{4}{|c|}{ ASA } & \multicolumn{4}{|c|}{ ASA log-transformed } \\
\hline Inter & 2.815 & 0.074 & 38.166 & $<0.001$ & 0.426 & 0.011 & 37.76 & $<0.001$ \\
\hline \multirow[t]{2}{*}{ Group } & 0.062 & 0.136 & 0.454 & 0.65 & 0.020 & 0.021 & 0.97 & 0.334 \\
\hline & \multicolumn{4}{|c|}{$\mathrm{FC}$} & \multicolumn{4}{|c|}{ FC log-transformed } \\
\hline Inter & 16.728 & 0.554 & 30.208 & $<0.001$ & 1.176 & 0.015 & 78.361 & $<0.001$ \\
\hline \multirow[t]{2}{*}{ Group } & -6.497 & 0.963 & -6.745 & $<0.001$ & -0.169 & 0.026 & -6.463 & $<0.001$ \\
\hline & \multicolumn{4}{|c|}{ RegNO } & \multicolumn{4}{|c|}{ RegNO log-transformed } \\
\hline Inter & 4.168 & 0.178 & 23.47 & $<0.001$ & 0.537 & 0.018 & 29.431 & $<0.001$ \\
\hline Group & -1.200 & 0.31 & -3.87 & $<0.001$ & -0.104 & 0.032 & -3.223 & 0.002 \\
\hline
\end{tabular}

2 
Table 6(on next page)

Table 6 Means and standard errors of eye movement measures on target words across conditions and age groups 
1 Table 6 Means and standard errors of eye movement measures on target words across conditions and age groups

\begin{tabular}{ccccccccc}
\hline \multirow{2}{*}{ measures } & \multicolumn{3}{c}{ Young adults readers } \\
\cline { 2 - 9 } & HF-P & LF-P & HF-U & LF-U & HF-P & LF-P & HF-U & LF-U \\
\hline FFD & $232(5.2)$ & $234(5.2)$ & $243(5.2)$ & $252(5.2)$ & $256(7.4)$ & $266(7.4)$ & $266(7.4)$ & $280(7.4)$ \\
DG & $254(8.7)$ & $261(8.7)$ & $275(8.7)$ & $299(8.7)$ & $302(12.3)$ & $342(12.3)$ & $336(12.3)$ & $399(12.3)$ \\
Go-Past & $349(15.9)$ & $364(15.9)$ & $447(15.9)$ & $458(15.9)$ & $365(22.5)$ & $454(22.5)$ & $467(22.5)$ & $527(22.5)$ \\
TRT & $287(18.8)$ & $314(18.8)$ & $399(18.8)$ & $437(18.8)$ & $367(26.6)$ & $447(26.6)$ & $534(26.6)$ & $605(26.6)$ \\
Skip.Pro & $24.9(1.8)$ & $24.4(1.8)$ & $21.1(1.8)$ & $20.8(1.8)$ & $11.8(2.6)$ & $12.0(2.6)$ & $12.8(2.6)$ & $8.7(2.6)$ \\
Refix.pro & $8.9(1.8)$ & $10.5(1.8)$ & $11.4(1.8)$ & $16.6(1.8)$ & $17.8(2.6)$ & $27.0(2.6)$ & $23.0(2.6)$ & $31.3(2.6)$ \\
ISL & $2.00(0.10)$ & $2.04(0.10)$ & $1.99(0.10)$ & $1.91(0.10)$ & $1.81(0.14)$ & $1.75(0.14)$ & $2.02(0.14)$ & $1.89(0.14)$ \\
OSL & $1.87(0.07)$ & $1.81(0.07)$ & $1.89(0.07)$ & $1.80(0.07)$ & $1.81(0.09)$ & $1.79(0.09)$ & $1.64(0.09)$ & $1.85(0.09)$ \\
\hline
\end{tabular}

2 (Note: Means and standard errors are computed across subjects' means, standard errors are shown in parentheses, time measures 3 are in milliseconds, probability measures are in \%, saccade length are in character spaces.) 
Table 7 (on next page)

Table 7 Linear mixed-effects model analyses on fixation time measures 
Table 7 Linear mixed-effects model analyses on fixation time measures

\begin{tabular}{|c|c|c|c|c|c|c|c|c|}
\hline \multicolumn{3}{|c|}{ FFD } & \multicolumn{6}{|c|}{ FFD log-transformed } \\
\hline & $b$ & $S E$ & $t$ & $p$ & $b$ & $S E$ & $t$ & $p$ \\
\hline Inter & 253.356 & 4.109 & 61.660 & $<0.001$ & 2.372 & 0.007 & 338.994 & $<0.001$ \\
\hline $\mathrm{F}$ & -8.917 & 2.896 & -3.079 & 0.002 & -0.015 & 0.005 & -2.97 & 0.003 \\
\hline $\mathrm{P}$ & -13.618 & 2.896 & -4.702 & $<0.001$ & -0.024 & 0.005 & -4.634 & $<0.001$ \\
\hline G & -27.459 & 7.397 & 3.712 & $<0.001$ & -0.052 & 0.012 & -4.221 & $<0.001$ \\
\hline $\mathrm{F} \times \mathrm{P}$ & 4.310 & 9.210 & 0.468 & 0.642 & 0.008 & 0.017 & 0.473 & 0.638 \\
\hline $\mathrm{F} \times \mathrm{G}$ & 6.421 & 5.791 & 1.109 & 0.268 & 0.006 & 0.010 & 0.610 & 0.542 \\
\hline $\mathrm{P} \times \mathrm{G}$ & -1.119 & 5.793 & -0.193 & 0.847 & -0.005 & 0.010 & -0.454 & 0.65 \\
\hline $\mathrm{P} \times \mathrm{F} \times \mathrm{G}$ & 0.419 & 11.585 & 0.036 & 0.971 & 0.004 & 0.021 & 0.209 & 0.834 \\
\hline \multicolumn{3}{|c|}{ GD } & \multicolumn{6}{|c|}{ GD log-transformed } \\
\hline & $b$ & $S E$ & $t$ & $p$ & $b$ & $S E$ & $t$ & $p$ \\
\hline Inter & 308.821 & 5.863 & 52.676 & $<0.001$ & 2.435 & 0.010 & 255.162 & $<0.001$ \\
\hline $\mathrm{F}$ & -35.235 & 4.913 & -7.171 & $<0.001$ & -0.037 & 0.006 & -6.115 & $<0.001$ \\
\hline$P$ & -38.56 & 4.914 & -7.846 & $<0.001$ & -0.045 & 0.006 & -7.363 & $<0.001$ \\
\hline G & -72.741 & 11.725 & -6.204 & $<0.001$ & -0.098 & 0.015 & -6.504 & $<0.001$ \\
\hline$F \times P$ & 19.22 & 9.827 & 1.956 & 0.051 & 0.019 & 0.026 & 0.732 & 0.469 \\
\hline $\mathrm{F} \times \mathrm{G}$ & 36.577 & 9.827 & 3.722 & $<0.001$ & 0.027 & 0.012 & 2.164 & 0.031 \\
\hline $\mathrm{P} \times \mathrm{G}$ & 16.512 & 9.829 & 1.680 & 0.093 & 0.009 & 0.012 & 0.77 & 0.442 \\
\hline \multirow[t]{3}{*}{$P \times F \times G$} & -7.645 & 19.654 & -0.389 & 0.697 & 0.012 & 0.024 & 0.494 & 0.621 \\
\hline & Go-past & & \multicolumn{6}{|c|}{ Go-past log-transformed } \\
\hline & $b$ & $S E$ & $t$ & $p$ & $b$ & $S E$ & $t$ & $p$ \\
\hline Inter & 428.841 & 15.106 & 28.389 & $<0.001$ & 2.542 & 0.012 & 205.264 & $<0.001$ \\
\hline $\mathrm{F}$ & -45.922 & 9.844 & -4.665 & $<0.001$ & -0.042 & 0.008 & -5.305 & $<0.001$ \\
\hline $\mathrm{P}$ & -93.263 & 9.847 & -9.472 & $<0.001$ & -0.081 & 0.008 & -10.171 & $<0.001$ \\
\hline G & -50.643 & 21.167 & -2.393 & 0.018 & -0.055 & 0.018 & -3.122 & 0.002 \\
\hline$F \times P$ & -18.555 & 47.399 & -0.391 & 0.698 & -0.015 & 0.038 & -0.398 & 0.693 \\
\hline $\mathrm{F} \times \mathrm{G}$ & 60.976 & 19.690 & 3.097 & 0.002 & 0.055 & 0.016 & 3.431 & $<0.001$ \\
\hline $\mathrm{P} \times \mathrm{G}$ & -5.006 & 19.694 & -0.254 & 0.799 & -0.007 & 0.016 & -0.447 & 0.655 \\
\hline \multirow[t]{3}{*}{$\mathrm{P} \times \mathrm{F} \times \mathrm{G}$} & 19.619 & 39.386 & 0.498 & 0.618 & -0.002 & 0.032 & -0.074 & 0.941 \\
\hline & TRT & & \multicolumn{6}{|c|}{ TRT log-transformed } \\
\hline & $b$ & $S E$ & $t$ & $p$ & $b$ & $S E$ & $t$ & $p$ \\
\hline Inter & 423.663 & 18.093 & 23.415 & $<0.001$ & 2.574 & 0.010 & 246.338 & $<0.001$ \\
\hline $\mathrm{F}$ & -53.903 & 9.032 & -5.968 & $<0.001$ & -0.043 & 0.008 & -5.510 & $<0.001$ \\
\hline$P$ & -140.059 & 9.032 & -15.507 & $<0.001$ & -0.114 & 0.008 & -14.551 & $<0.001$ \\
\hline G & -128.83 & 27.043 & -4.764 & $<0.001$ & 0.097 & 0.021 & -4.648 & $<0.001$ \\
\hline $\mathrm{F} \times \mathrm{P}$ & 0.326 & 51.371 & 0.006 & 0.995 & -0.011 & 0.016 & -0.724 & 0.469 \\
\hline $\mathrm{F} \times \mathrm{G}$ & 42.285 & 18.065 & 2.341 & 0.019 & 0.025 & 0.016 & 1.579 & 0.114 \\
\hline $\mathrm{P} \times \mathrm{G}$ & 45.491 & 18.065 & 2.518 & 0.012 & 0.017 & 0.016 & 1.117 & 0.264 \\
\hline $\mathrm{P} \times \mathrm{F} \times \mathrm{G}$ & 19.5525 & 36.129 & 0.541 & 0.588 & 0.017 & 0.031 & 0.539 & 0.59 \\
\hline
\end{tabular}

2 (Note. $\mathrm{F}=$ frequency factors; $\mathrm{P}=$ predictable factors; $\mathrm{G}=$ group factors)

3 


\section{Table 8 (on next page)}

Table 8 Linear mixed-effects model analyses on probability and saccade measures 
1 Table 8 Linear mixed-effects model analyses on probability and saccade measures

\begin{tabular}{|c|c|c|c|c|c|c|c|c|}
\hline & \multicolumn{4}{|l|}{ Skipping } & \multicolumn{4}{|c|}{ Re-fixating } \\
\hline & $b$ & $S E$ & $Z$ & $p$ & $b$ & $S E$ & $Z$ & $p$ \\
\hline Inter & -1.953 & 0.112 & -17.372 & $<0.001$ & -1.945 & 0.135 & -14.4 & $<0.001$ \\
\hline $\mathrm{F}$ & 0.128 & 0.094 & 1.354 & 0.176 & -0.459 & 0.087 & -5.275 & $<0.001$ \\
\hline $\mathrm{P}$ & 0.192 & 0.094 & 2.041 & 0.041 & -0.373 & 0.087 & -4.294 & $<0.001$ \\
\hline G & 1.137 & 0.206 & 5.524 & $<0.001$ & -0.948 & 0.222 & -4.262 & $<0.001$ \\
\hline $\mathrm{P} \times \mathrm{F}$ & -0.253 & 0.247 & -1.025 & 0.305 & 0.086 & 0.339 & 0.254 & 0.799 \\
\hline $\mathrm{F} \times \mathrm{G}$ & -0.200 & 0.188 & -1.059 & 0.290 & 0.238 & 0.174 & 1.373 & 0.17 \\
\hline$P \times G$ & 0.080 & 0.188 & 0.426 & 0.67 & -0.125 & 0.173 & -0.723 & 0.47 \\
\hline$P \times F \times G$ & 0.512 & 0.377 & 1.359 & 0.174 & 0.426 & 0.347 & 1.229 & 0.219 \\
\hline \multicolumn{3}{|c|}{ ISL } & \multicolumn{6}{|c|}{ ISL log-transformed } \\
\hline & $b$ & $S E$ & $t$ & $p$ & $b$ & $S E$ & $t$ & $p$ \\
\hline Inter & 1.922 & 0.078 & 24.618 & $<0.001$ & 0.214 & 0.015 & 14.392 & $<0.001$ \\
\hline $\mathrm{F}$ & -0.059 & 0.037 & -1.597 & 0.110 & 0.007 & 0.007 & 1.066 & 0.287 \\
\hline $\mathrm{P}$ & 0.031 & 0.037 & -0.837 & 0.403 & -0.0003 & 0.007 & -0.047 & 0.962 \\
\hline $\mathrm{G}$ & -0.121 & 0.150 & -0.830 & 0.408 & 0.016 & 0.027 & 0.596 & 0.553 \\
\hline $\mathrm{P} \times \mathrm{F}$ & -0.081 & 0.133 & -0.611 & 0.545 & -0.022 & 0.030 & -0.747 & 0.459 \\
\hline $\mathrm{F} \times \mathrm{G}$ & -0.038 & 0.073 & -0.524 & 0.601 & 0.002 & 0.014 & 0.165 & 0.869 \\
\hline $\mathrm{P} \times \mathrm{G}$ & 0.219 & 0.073 & 2.992 & 0.003 & 0.030 & 0.014 & 2.173 & 0.030 \\
\hline $\mathrm{P} \times \mathrm{F} \times \mathrm{G}$ & 0.061 & 0.147 & 0.414 & 0.679 & -0.065 & 0.027 & -2.370 & 0.018 \\
\hline \multicolumn{3}{|c|}{ OSL } & \multicolumn{6}{|c|}{ OSL log-transformed } \\
\hline & $b$ & $S E$ & $t$ & $p$ & $b$ & $S E$ & $t$ & $p$ \\
\hline Inter & 1.793 & 0.057 & 31.696 & $<0.001$ & 0.209 & 0.012 & 17.059 & $<0.001$ \\
\hline $\mathrm{F}$ & 0.011 & 0.034 & 0.316 & 0.752 & 0.014 & 0.006 & 2.441 & 0.015 \\
\hline $\mathrm{P}$ & 0.04 & 0.034 & 1.190 & 0.234 & 0.020 & 0.006 & 3.603 & $<0.001$ \\
\hline G & 0.079 & 0.095 & 0.833 & 0.407 & 0.025 & 0.028 & 1.177 & 0.242 \\
\hline $\mathrm{P} \times \mathrm{F}$ & 0.101 & 0.141 & 0.716 & 0.478 & 0.020 & 0.028 & 0.723 & 0.474 \\
\hline $\mathrm{F} \times \mathrm{G}$ & 0.148 & 0.067 & 2.201 & 0.028 & 0.023 & 0.011 & 2.102 & 0.036 \\
\hline$P \times G$ & -0.081 & 0.067 & -1.205 & 0.228 & -0.014 & 0.011 & -1.295 & 0.195 \\
\hline $\mathrm{P} \times \mathrm{F} \times \mathrm{G}$ & 0.264 & 0.134 & 1.956 & 0.051 & -0.063 & 0.022 & -2.82 & 0.005 \\
\hline
\end{tabular}

(Note. $\mathrm{F}=$ frequency factors; $\mathrm{P}=$ predictable factors; $\mathrm{G}=$ group factors) 